\title{
El uso de un modelo interaccionista para el análisis de prácticas docentes
}

The use of an interactionist model for the teaching practice analysis

\section{Volumen 18, Número 3 \\ Setiembre-Diciembre}

pp. $1-20$

Este número se publica el 1 de setiembre de 2018

DOI: https://doi.org/10.15517/aie.v18i3.34381

\author{
Ariana Fragoza González \\ Graciela Cordero Arroyo \\ Cecilia Fierro Evans
}

Revista indizada en REDALYC, SCIELO

Revista distribuida en las bases de datos:

LATINDEX, DOAJ, REDIB, IRESIE, CLASE, DIALNET, SHERPA/ROMEO, QUALIS-CAPES, MIAR

Revista registrada en los directorios:

ULRICH'S $, \underline{R E D I E}, \underline{R I N A C E}, \underline{\text { OEI }}, \underline{\text { MAESTROTECA }}, \underline{\text { PREAL, }}$ 


\title{
El uso de un modelo interaccionista para el análisis de prácticas docentes
}

The use of an interactionist model for the teaching practice analysis

\author{
Ariana Fragoza González ${ }^{1}$ \\ Graciela Cordero Arroyo ${ }^{2}$ \\ Cecilia Fierro Evans ${ }^{3}$
}

\begin{abstract}
Resumen: El presente ensayo tiene la finalidad de describir el Modelo E-P-R (Epistemológica-PragmáticaRelacional). Este modelo es un referente teórico-metodológico utilizado para la formación de docentes, desde una perspectiva reflexiva. Su autora principal es Isabelle Vinatier. El modelo E-P-R se sustenta en elementos teóricos que provienen de las disciplinas psicológica y sociológica. Para el análisis de la práctica docente, el modelo E-P$R$ aporta tres categorías teóricas, que refieren a las dimensiones presentes en la práctica docente de forma simultánea: la Epistemológica, la Pragmática y la Relacional (responden a las siglas E-P-R del modelo). El ejercicio reflexivo acerca de la propia práctica docente da pauta para profundizar en cuestionamientos como: ¿cómo se han construido las acciones que se llevan a cabo?, ¿por qué se hacen las cosas de esta manera y no de otra? y ¿qué significado tiene para el propio docente? Esta serie de preguntas pueden marcar la pauta para que la persona docente mejore su práctica a partir de hacerse sujeto consciente sobre su propio actuar. En México, las experiencias formativas con docentes de educación básica que hacen uso del modelo $E-P-R$ evidencian que el modelo fortalece a las personas docentes como sujetos, como profesionales competentes, aportando una mirada autoevaluativa y crítica sobre su práctica y la de sus colegas.
\end{abstract}

Palabras clave: formación de docentes, práctica pedagógica, interacción social, aprendizaje mediante la práctica

\begin{abstract}
The present essay has the purpose of describing the E-P-R Model (Epistemological-PragmaticRelational). This model is a theoretical-methodological reference used for the training of teachers, from a reflective perspective. The main author is Isabelle Vinatier. The E-P-R model is based on theoretical elements that come from the psychological and sociological discipline. For the analysis of teaching practice, the E-P-R model provides three theoretical categories, which refer to the dimensions present in the teaching practice simultaneously: the Epistemological, Pragmatic and Relational (responds to the acronym E-P-R of the model). The reflexive exercise about the teaching practice gives guidelines to deepen questions such as: How have the actions been carried out? Why are things done this way and not another? and What does it mean for the teacher? This series of questions can set the tone for the teacher to improve their practice by becoming a conscious subject about their own actions. In Mexico, training experiences with basic education teachers using the EPR model evidenced that the model strengthens teachers as subjects, as competent professionals, providing a self-evaluating perspective and criticism about his practice and that of his colleagues.
\end{abstract}

Key Words: teacher education, teaching practice, social interaction, experience training

\footnotetext{
1 Investigadora de la Universidad Autónoma de Baja California, Instituto de Investigación y Desarrollo Educativo, México. Maestría en Ciencias Educativas. Dirección electrónica: ariana.fragoza@uabc.edu.mx

2 Investigadora de la Universidad Autónoma de Baja California, Instituto de Investigación y Desarrollo Educativo, México. Dirección electrónica: gcordero@uabc.edu.mx

3 Investigadora de la Universidad Iberoamericana León, México. Dirección electrónica: cecilia.fierro@iberoleon.mx
}

Ensayo recibido: 23 de marzo, 2018

Enviado a corrección: 26 de junio, 2018

Aprobado: 13 de agosto, 2018 


\section{Introducción}

El presente ensayo tiene la finalidad de describir el modelo E-P-R (EpistemológicaPragmática-Relacional) desarrollado en Europa desde hace más de una década por Isabelle Vinatier (2013), miembro de la Observation de Practiques Éducatives et Enseignantes (OPÉEN, por sus siglas en francés) para analizar prácticas docentes ${ }^{4}$. El modelo E-P-R se deriva de los planteamientos interaccionistas desarrollados por Marguerite Altet y la propia Isabelle Vinatier en 2008.

El modelo E-P-R es un referente teórico-metodológico que funciona como un dispositivo de formación continua de docentes desde un enfoque reflexivo, a partir de la profundización sobre la propia práctica en situación de aula. Tiene sus bases teóricas en la psicología y la sociología. Desde ambas perspectivas disciplinarias, Vinatier (2013) retoma elementos como el de la influencia de la interacción social para el desarrollo del aprendizaje, la importancia de las estructuras mentales del sujeto, las realidades construidas en interacción y los significados compartidos.

Este modelo ha tenido influencia en Europa, África y, recientemente, en Latinoamérica. En México, el modelo E-P-R ha sido abordado y utilizado por Cecilia Fierro y Bertha Fortoul en diversas obras (2014; 2015; 2016 y 2017). Asimismo, en Argentina, se han tomado planteamientos interaccionistas por Pereyra, Moscato, Calderón y Oviedo (2016). Dado que se cuenta con poco material en español sobre el modelo E-P-R (Paquay, Altet, Charlier y Perrenoud, 2010), los autores antes mencionados han hecho revisiones importantes para la mejor comprensión del modelo en América Latina.

Este ensayo se divide en dos grandes apartados. En el primero se describen las corrientes teóricas que forman las bases conceptuales del modelo E-P-R. Se explican brevemente dos corrientes teóricas pertenecientes a la disciplina psicológica: la didáctica profesional, desarrollada por Pierre Pastré, y la teoría de los campos conceptuales, construida por Gérard Vergnaud. Además, se recuperan dos corrientes teóricas pertenecientes a la sociología: el interaccionismo simbólico de Herbert Blumer y el constructivismo social, planteado por Peter Berger y Thomas Luckmann. En el segundo apartado del artículo se hace una descripción del modelo E-P-R, la importancia de la reflexión como oportunidad para mejorar las prácticas docentes, a partir de la identificación de las reglas de acción docente y sus principios pedagógicos.

\footnotetext{
${ }^{4}$ Entendidas como las acciones que se llevan a cabo dentro del aula, y que están relacionadas con el proceso de enseñanza (De Lella, 1999).
} 


\section{Corrientes teóricas que nutren el modelo E-P-R}

\subsection{Perspectiva psicológica}

La didáctica profesional desarrollada por Pierre Pastré y la teoría de los campos conceptuales de Gerard Vergnaud son consideradas como corrientes que nutren el modelo E-P-R, debido a los elementos que estas aportan a su construcción. A continuación, se describen ambas teorías.

\subsubsection{La didáctica profesional}

La didáctica profesional es una corriente teórica desarrollada por el francés Pierre Pastré (2002; 2006; 2007 y 2011). La didáctica profesional tiene el objetivo de analizar la actividad profesional manifestada en la profesión docente (Pastré, 2007). De esta forma, el maestro cuenta con un dispositivo de análisis de la propia práctica en acto, en un marco en el que la diversidad de tareas hace que el análisis de las actividades sea complejo. La didáctica profesional permite identificar la conceptualización que la persona docente otorga a diversas acciones y saberes, así como la forma de operar en el aula, lo cual define la construcción que ha realizado de su propia práctica a través de la experiencia (Moscato, 2016).

Por su parte, Pastré (2002) explica que las acciones eficaces que se presentan en el aula, generalmente, tienden a mostrar variaciones e irregularidades. Sin embargo, las acciones también pueden presentar capacidad para adaptarse a las circunstancias y pueden ser flexibles de acuerdo con las necesidades particulares del estudiantado o el cuerpo docente. En este sentido, las acciones eficaces suelen organizarse en un ambiente variante (Pastre, 2002, citado en Moscato, 2016).

\subsubsection{Teoría de los campos conceptuales}

Gerárd Vergnaud (1990) desarrolló la teoría de los campos conceptuales en 1990. Toma como referente directo la teoría cognoscitiva de Jean Piaget. La teoría de Vergnaud aporta al campo de la didáctica profesional tres grandes constructos: esquema, situación y experiencia. Desde los planteamientos de Piaget, los esquemas "son las unidades de organización que posee el sujeto cognoscente" (Hernández, 2012, p.178). Sobre este tema, Hernández expone, a partir de una analogía, que los esquemas mentales son los ladrillos de la construcción del sistema intelectual y cognitivo. Un esquema está compuesto por reglas de actuación y anticipaciones, ya que las acciones generalmente buscan logar un objetivo. A su vez, estas acciones realizadas están compuestas de invariantes operatorias, es decir, que al 
actuar el sujeto generalmente se encuentra con situaciones que provocan variaciones en su actuar, por lo que tiene que estar constantemente ajustándose a las circunstancias (Vergnaud, 1990).

Los esquemas mentales pueden clasificarse por medio de campos conceptuales. Un campo conceptual es un conjunto de situaciones que llevan al sujeto a utilizar un esquema mental. Por ejemplo: un campo conceptual de las estructuras aditivas referiría a un conjunto de situaciones que requieren la realización de una adición (Vergnaud, 1990). Los campos conceptuales permiten la organización de los esquemas y estructuras mentales.

Un segundo concepto abordado en la teoría de los campos conceptuales es el de situación. El concepto de situación ha sido definido más claramente por Guy Brousseau (2007), quien plantea que tanto la situación como los procesos cognitivos y las respuestas del sujeto son producto de las situaciones en las cuales el sujeto se ve involucrado. Las situaciones, a su vez, se pueden conceptualizar de acuerdo con dos lógicas (Brousseau, 2007):

a) La lógica de la variedad: en un campo conceptual existe una gran variedad de situaciones, las variables que puedan presentarse en cada situación son un medio para desarrollar sistemáticamente diferentes clases de situaciones.

b) La lógica de la historia: los conocimientos que poseen los estudiantes son determinados a partir de las situaciones en las que se han encontrado (experiencias) y dominado progresivamente.

Un tercer concepto desarrollado por Vergnaud es el de experiencia. Este concepto es definido claramente por Vinatier (2013) como aquello que implica aumentar las posibilidades de acción y enriquecer los conocimientos a través del contacto con la realidad y la vida diaria. La experiencia es variable, ya que se ve influida constantemente por contingencias determinadas por el contexto en el que se practica.

La teoría de los campos conceptuales ha contribuido a la construcción conceptual y teórica del campo de la didáctica profesional y del modelo de Vinatier (2013). Los conceptos de esquema, experiencia y situación son elementos clave para la comprensión de la práctica docente en situación. Los elementos conceptuales definidos por Vergnaud (1990) aportan a la conceptualización y análisis de lo que hace y dice la persona docente en el aula. 


\subsection{Perspectiva sociológica}

También, se consideran antecedentes del modelo E-P-R, la teoría social del interaccionismo simbólico desarrollada por Herbert Blumer y la teoría del constructivismo social desarrollada por Peter Berger y Thomas Luckmann. Ambas corrientes se explicarán a continuación.

\subsubsection{Interaccionismo simbólico}

El interaccionismo simbólico desarrollado por Blumer (1969) parte de tres premisas: (1) los seres humanos actúan hacia las cosas sobre la base de los significados que las cosas tienen para ellos; (2) el significado de las $\operatorname{cosas}^{5}$ se deriva o surge de la interacción social que uno tiene con sus semejantes; y (3) estos significados se manejan y se modifican a través de un proceso interpretativo utilizado por la persona para tratar con las cosas que encuentra. El interaccionismo simbólico de Blumer (1969) plantea que los sentidos de ciertas conductas se significan mediante la interacción social. El conjunto de significaciones derivadas de la interacción entre sujetos da como resultado un conjunto de símbolos que, para los actores involucrados en la interacción, tienen un sentido y para otros sujetos puede no tenerlo.

Por medio del lenguaje, los sujetos pueden interactuar e intercambiar símbolos, tanto verbales como no verbales. Cuando se ha comunicado un símbolo que tiene sentido para el emisor y para el receptor, se puede decir que es un símbolo significativo. Por medio de los símbolos significativos, el sujeto puede predecir las acciones de los demás, a través de los significados que se han compartido con anterioridad (Blumer, 1969). El interaccionismo simbólico cuenta con un concepto clave, el objeto. El objeto es una cosa que adquiere un significado gracias a la actuación que hacen los sujetos en torno a él. Si el objeto no tiene significado por sí mismo, al otorgarle un significado y al actuar en torno a él, este va adquiriendo riqueza conceptual. De esta manera, los sujetos tienen una perspectiva más amplia de lo que significa un objeto, gracias a las interacciones que se dan en torno a él (Blumer, 1969).

Dialogar con los demás tiene un fin en sí mismo, que es establecer relaciones sociales. El diálogo permite no solo encontrar significados a las acciones de los demás, es decir, significados internos a la persona, sino que, además, el diálogo busca predecir los significados y las acciones de otras personas. Estas predicciones en el actuar del prójimo

${ }^{5}$ Entendidas por Blumer (1969) como todos los objetos, tanto del mundo físico como del mundo abstracto. 
pueden llevar al sujeto a establecer interacciones que enriquezcan el conocimiento (Blumer, 1969).

\subsubsection{La construcción social de la realidad}

Berger y Luckmann (1968) exponen que la realidad se construye socialmente. La sociología del conocimiento es la encargada de estudiar el proceso de la construcción de esa realidad particular. Tanto el concepto de realidad como el de conocimiento se asocian con la interacción social, ya que por medio de la interacción se obtienen experiencias sobre una realidad particular, las cuales enriquecen el aprendizaje de los individuos. La realidad es aquello que se comparte con los otros. En este sentido, ¿cómo experimentamos la vida cotidiana con los demás? La realidad se experimenta al entrar en situaciones cara a cara, esto es, a través de la interacción social.

En la situación "cara a cara" el otro se me aparece en un presente vivido que ambos compartimos. Sé que en el mismo presente vivido yo me le presento a él. Mi "aquí y ahora" y el suyo gravitan continuamente uno sobre otro, en tanto dure la situación "cara a cara". El resultado es un intercambio continuo entre mi expresividad y la suya. (Berger y Luckmann, 1968, p. 46)

Como lo plantean Berger y Luckmann (1968) la realidad es algo que se comparte con los demás en la vida cotidiana, a través del lenguaje, ya sea verbal o no verbal. El lenguaje, entonces, juega un papel primordial para la interacción social y para la adquisición de conocimiento. Berger y Luckmann dan un ejemplo en relación con este planteamiento: al entrar en interacción con otro, pueden darse expresiones no verbales como sonreír. Cuando una persona le sonríe a otra, y esta última responde con un ceño fruncido, la primera persona deja de sonreír. El ejemplo descrito da una visión de las interpretaciones que se hacen cotidianamente del lenguaje verbal y no verbal, y que para los sujetos puede convertirse en un aprendizaje.

El conocimiento se obtiene a partir de la interacción cara a cara en una realidad cotidiana, y este se acumula como un tipo de acopio social, esto quiere decir que son cuerpos de conocimiento compartidos con otros. Un ejemplo de lo anterior es:

Sé que soy pobre y que, por lo tanto, no puedo pretender vivir en un barrio elegante. Este conocimiento lo comparto, claro está, con aquellos que también son pobres y con aquellos que gozan de una situación más privilegiada. De esta manera, la participación 
en el cúmulo social de conocimiento permite la "ubicación" de los individuos en la sociedad y el "manejo" apropiado de ellos (Berger y Luckmann, 1968, p. 60).

A partir de este ejemplo, se puede decir que los cuerpos de conocimiento compartidos por la sociedad juegan un papel importante para darle cierta identidad a aquello que nos rodea. Así pues, la interacción entre sujetos abre una ventana al conocimiento, a partir de que los cuerpos de conocimiento son interpretados o entendidos de forma distinta por cada persona, ya que su propia identidad e historia determinan su forma de ver e interpretar la realidad.

\section{El modelo E-P-R para analizar las prácticas docentes}

Como se describió anteriormente, el modelo E-P-R tiene sus raíces conceptuales en dos disciplinas distintas. Los conceptos recuperados de cada una ellas se muestran en la figura 1.

Figura 1

Conceptos de los que se nutre el modelo E-P-R

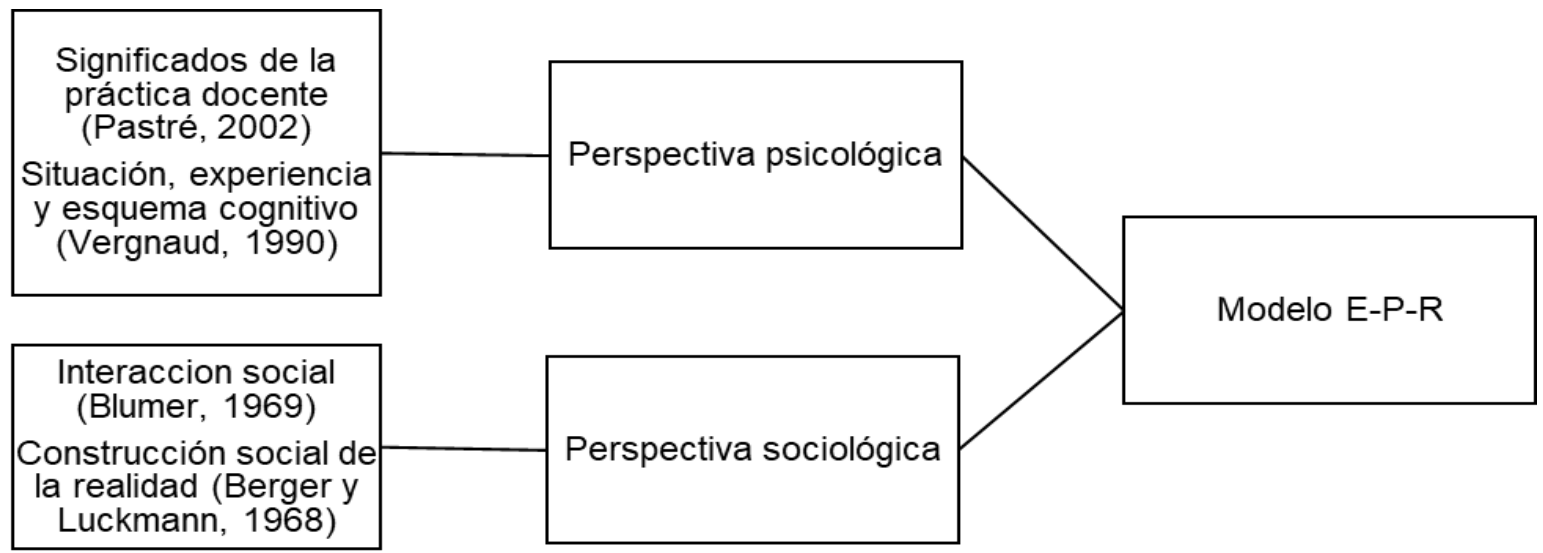

Fuente: Elaboración propia, a partir de lo planteado por Pastré (2002; 2006; 2007 y 2011), Vergnaud (1990), Blumer (1969) y Berger y Luckmann (1968).

El modelo E-P-R estudia la práctica docente en el aula a partir de la intersubjetividad, entendida como un elemento central para analizar y comprender la comunicación establecida entre la persona docente y el estudiantado, en torno a los contenidos que se van a abordar o a tareas más específicas que requieren del intercambio lingüístico verbal o no verbal. Así pues, el análisis de la intersubjetividad del sujeto permite dar cuenta de cómo la persona 
docente actúa y organiza sus ideas de forma simultánea para responder a las necesidades que se presentan en el momento de la práctica.

El modelo propuesto por Vinatier (2013) reconoce que las escuelas se encuentran inmersas en un entorno donde influyen decisiones políticas, sociales, económicas y culturales. Esta influencia se ve reflejada en la construcción de las tareas a realizar por cada actor educativo, los cambios organizacionales de las escuelas, la vinculación que se forma con organizaciones externas y con los padres de familia, entre otros. La influencia constante de agentes externos a la escuela, en diversas ocasiones determina la tarea de quien enseña y la forma en que este es objeto de contantes revisiones por parte de las autoridades educativas.

El modelo E-P-R también reconoce al docente y a la docente docente como personas, lo cual permite abrir un abanico de situaciones que les rodean, situaciones contextuales que influyen directamente en su práctica y en su persona. De esta forma, se evidencia que la persona docente tiene una tarea compleja, en la cual la práctica en el aula puede dar giros que no espera y situaciones emergentes en las que debe actuar con la mayor asertividad posible.

La práctica docente se desarrolla en interacción, entre el o la docente, la comunidad estudiantil y entre las mismas personas estudiantes (Vinatier y Altet, 2008). Las interacciones áulicas se construyen a partir de episodios o "ciclos" de intercambios, a partir de los quehaceres y decires de quienes participan, de las interpretaciones y de las reacciones o acciones ajustadas. Seguidamente, se presenta la Figura 2, en la cual se muestra, de manera gráfica, cómo se constituyen los ciclos de intercambios entre dos o más actores. 
Figura 2

Ciclo de la interacción áulica

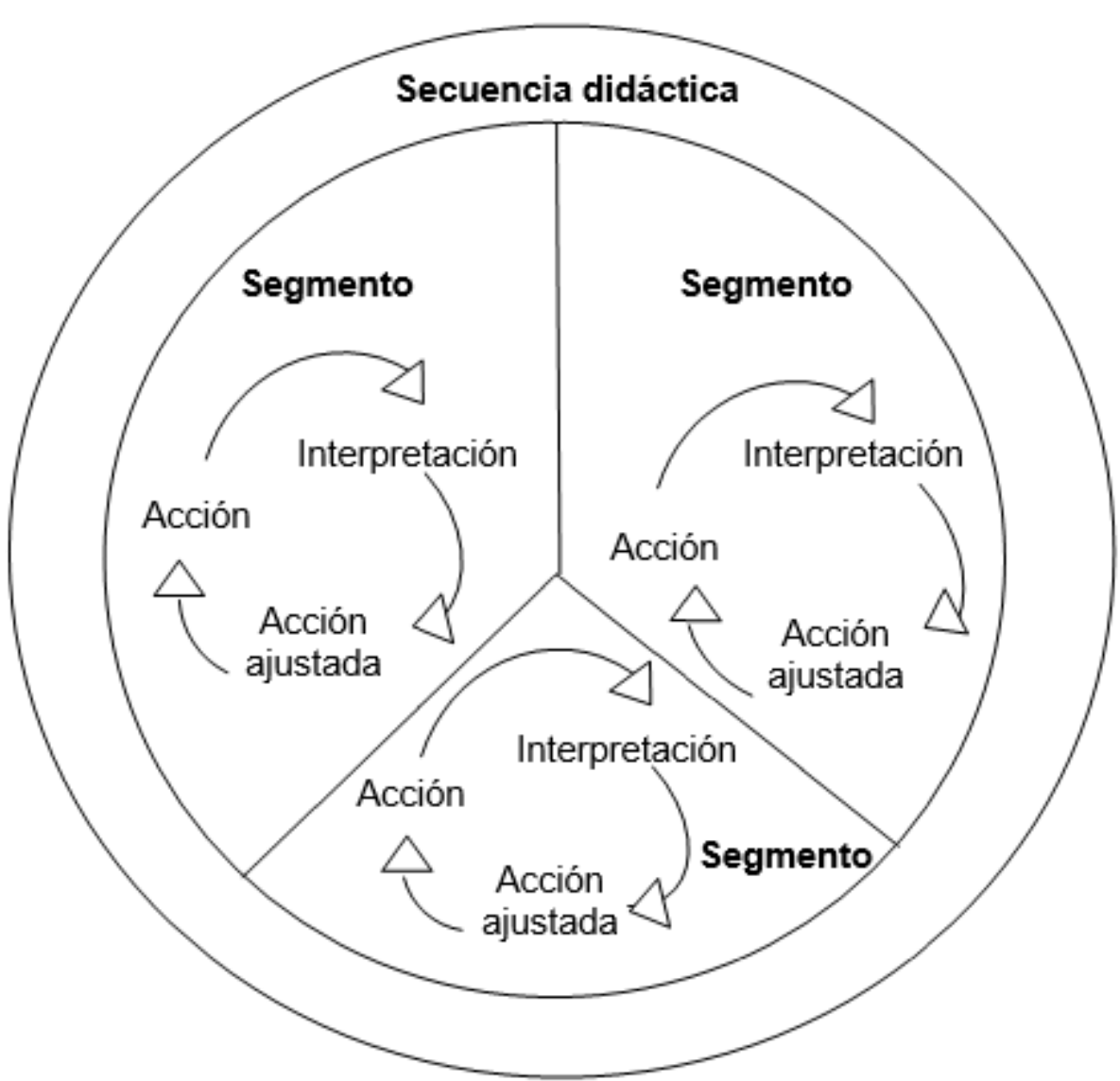

Fuente: Adaptado de "Interacción en el espacio del salón de clase" por Fierro, y Fortoul (2014). La gestión de los aprendizajes para la convivencia y para las disciplinas en las prácticas docentes, como objeto de estudio.

Como se puede observar en la figura anterior, las interacciones entre docentes y estudiantes se forman de acciones que se realizan a partir de un conocimiento adquirido. Al interpretar estas acciones influyen las creencias, emociones y motivaciones de ambos actores. La interpretación del estudiantado genera que la persona docente ajuste su acción, de la cual se vuelve a generar una interpretación y así consecutivamente. Mediante los ciclos de interacciones en el aula, se logra la construcción del actuar de la persona docente con el actuar de cada uno de sus estudiantes. 


\subsection{El análisis de la práctica docente desde la perspectiva del modelo E-P-R}

El análisis de la práctica docente realizada desde la mirada del modelo E-P-R requiere de categorías teóricas en las cuales sustentarse, ya que el análisis de la práctica docente no puede realizarse de forma improvisada debido a la precisión que se necesita para el manejo de la información derivada del análisis. Estas categorías teóricas están constituidas por tres lógicas que intervienen simultáneamente en el aula, y que, por lo mismo, se tornan en polos de tensión que la persona docente ha de resolver en cada situación de aula. Cada una de estas lógicas se explican a continuación (Vinatier, 2013):

\subsubsection{Lógica epistemológica}

La lógica epistemológica da cuenta de las situaciones en las que el sujeto opera saberes interiorizados, estos se mantienen ajenos hasta que el objeto del mundo de la vida es captado, adaptado y apropiado. Estas acciones que dan cuenta del saber se exteriorizan a través de un discurso reflexivo donde tratan de evidenciar los rompimientos epistemológicos a los que el sujeto ha llegado (Yurén, 2008, citado en Fierro y Fortoul, 2017). En otras palabras, esta lógica refiere la construcción de los saberes, particularmente, aquellas interacciones que se dan en torno al contenido visto en clase.

Charlot (2006, citado en Fierro y Fortoul, 2017) plantea que la adquisición del saber se puede distinguir de acuerdo con tres figuras que aluden a lo objetivo, lo subjetivo y lo social, y que se articulan entre sí:

- La apropiación de un saber-objeto: refiere un saber materializado que se hace presente por medio de objetos externos al sujeto, como el saber adquirido por medio de materiales escritos o audiovisuales. A partir de este saber materializado, el sujeto puede formar un discurso descriptivo, explicativo y argumentativo.

- El dominio de una actividad, de una situación o de un objeto: hace referencia a la operacionalización del saber aprendido del objeto materializado, es decir, a las actividades realizadas por el sujeto, que surgen a partir del conocimiento del objeto.

- La apropiación de dispositivos relacionales: alude al aprendizaje subjetivo, que refiere el conocimiento de sí mismo, lo cual permite la construcción de una identidad personal y social. Los sentimientos y emociones en acto permiten, al mismo tiempo, relacionarse con el otro, lo que permite la construcción de un saber socializado. 


\subsubsection{Lógica pragmática}

Hace referencia a la utilización que hace el docente de herramientas metodológicas para orientar la construcción de los aprendizajes en el aula. Se consideran los objetivos de aprendizaje, las estrategias y las técnicas que gestiona la persona docente para acercar al o la estudiante al conocimiento. Un ejemplo de esta dimensión es la utilización de la pregunta como estrategia para rescatar conocimientos previos sobre un nuevo contenido.

La lógica pragmática de la práctica es la más cercana al cuerpo docente que recién ingresa al servicio, debido a que la experiencia es escasa y el o la docente debe de partir de la aplicación de un abanico de herramientas metodológicas hasta encontrar las que se adecuen mejor con su forma de trabajo.

\subsubsection{Lógica relacional}

Esta lógica se centra en la comunicación en el aula, se visualiza como un intercambio continuo entre la persona docente y sus estudiantes y entre el estudiantado mismo, donde intervienen las decisiones de los sujetos, sus emociones, sus motivaciones, etc. La finalidad de esta comunicación es la construcción de las condiciones para disponerse al aprendizaje, así como el manejo de la dinámica que se establece entre estudiantes y docentes.

Las tres lógicas intervienen simultáneamente en la práctica docente, lo que la vuelve compleja. Esta complejidad recae en la necesidad de satisfacer y trabajar cada una de las lógicas en el aula de clases, tratando de mantener un equilibrio constante entre ellas. EI equilibrio entre las tres lógicas es siempre inestable, por lo que como se señaló, las lógicas se convierten en polos de tensión en el momento en el que cada uno tiene que ser atendido de igual manera por el o la docente durante su intervención en el aula.

La falta de equilibrio entre los polos de tensión es normal, ya que la persona docente tiene que cumplir con tres funciones: enseñar el contenido, implementar estrategias didácticas y mantener una comunicación con sus estudiantes, lo cual en situaciones reales es difícil de gestionar de igual forma. La inestabilidad de los tres polos de tensión tiene su razón de ser en el hecho de que operan de manera concomitante; además, de la influencia del contexto, las actuaciones del estudiantado y las situaciones imprevistas en la cotidianidad del aula (Vinatier y Altet, 2008; Vinatier, 2013 y 2015, citadas en Fierro y Fortoul, 2015).

El abordaje de la forma en que se desenvuelven estos polos de tensión supone formular preguntas de tipo descriptivo, como las que se muestran en la Tabla 1: 
Tabla 1

Ejemplos de preguntas para describir la práctica docente por polo de tensión.

\begin{tabular}{|c|c|c|}
\hline Polo relacional & Polo pragmático & Polo epistemológico \\
\hline $\begin{array}{l}\text { ¿Qué rasgos definen las formas } \\
\text { de trato que se observan entre } \\
\text { estudiantes y docentes? } \\
\text { (distensión, burla, lenguaje } \\
\text { hostil, escucha atenta, cercanía) } \\
\text { ¿Se presentan eventos } \\
\text { disruptivos? ¿Con qué } \\
\text { frecuencia e intensidad? } \\
\text { ¿Qué normas y acuerdos de } \\
\text { trabajo se hacen evidentes o } \\
\text { son aludidos en el curso de la } \\
\text { clase? }\end{array}$ & $\begin{array}{l}\text { ¿Qué propósitos se aprecian a } \\
\text { partir de las actividades que } \\
\text { hace el estudiantado? } \\
\text { ¿Qué tipo de intervenciones } \\
\text { docentes guían la secuencia de } \\
\text { la clase? (centradas en el } \\
\text { contenido, en el o la estudiante } \\
\text { o en la organización de la } \\
\text { actividad) } \\
\text { ¿Qué materiales, instrumentos o } \\
\text { recursos de apoyo ofrece la } \\
\text { persona docente al } \\
\text { estudiantadodurante actividades los } \\
\text { ejercicios, ac, action } \\
\text { dinámicas grupales? }\end{array}$ & $\begin{array}{l}\text { ¿Qué hacen la persona docente } \\
\text { y el estudiantado con la } \\
\text { información? (se retoma sin } \\
\text { elaborar, se analiza, se } \\
\text { cuestiona, se reelabora, se } \\
\text { presentan otras situaciones, } \\
\text { etc.) dónde proviene la } \\
\text { ¿De ce do la } \\
\text { información con la que están } \\
\text { trabajando? (conocimiento de } \\
\text { sentido común, de la } \\
\text { experiencia, de los saberes } \\
\text { personales, familiares o } \\
\text { comunitarios o el conocimiento } \\
\text { especializado) } \\
\text { ¿Qué hace la persona docente } \\
\text { frente a las diferencias de nivel } \\
\text { mostrado por el estudiantado en } \\
\text { la apropiación del saber-obieto? }\end{array}$ \\
\hline
\end{tabular}

Fuente: Adaptado de Fierro y Fortoul (2017). Entretejer espacios para aprender y convivir en el aula (pp. 82-88). México: Ediciones SM.

Este tipo de preguntas, con fines descriptivos, darán lugar a una comprensión progresivamente más profunda de lo que acontece en el aula por parte del cuerpo docente.

\subsection{Reglas de acción y principios pedagógicos que guían la práctica docente}

La práctica docente en el aula se caracteriza por presentar recurrencias en el momento del actuar. Esto quiere decir que la persona docente, a partir de la experiencia y las situaciones en las que ha sido partícipe, forma patrones de comportamiento que reflejan la manera en que interpreta y actúa en situaciones cotidianas. Las recurrencias muestran la formación desarrollada por la experiencia en el aula. A los patrones observados en la práctica docente se les denomina reglas de acción. Las reglas de acción son definidas como:

Los patrones relativamente estables identificados en las prácticas docentes que reflejan la manera peculiar en que los docentes interpretan y enfrentan las situaciones cotidianas. Son las formas recurrentes de actuación de los docentes para atender cualquiera de los polos de tensión o para buscar un equilibrio entre ellos. (Fierro y Fortoul, 2017, p.100) 
En definitiva, cada docente presenta reglas de acción diferentes, ya que las experiencias vividas a lo largo de la práctica varían para cada uno. A partir de las reglas de acción se infieren los principios pedagógicos que guían la actuación docente. Vinatier y Altet (2008, citados en Fierro y Fortoul, 2017) definen los principios pedagógicos como aquellos razonamientos, creencias y teorizaciones, implícitas o explícitas que dan sentido y coherencia a las acciones docentes en el aula. Además, los principios pedagógicos son aquellos supuestos teórico-metodológicos que la persona docente considera que son verdaderos o adecuados para su trabajo.

Los principios pedagógicos han sido construidos por la persona docente a lo largo de su práctica y le dotan de significado a lo que hace en el aula. Los principios pedagógicos corresponden a la combinación entre las características consideradas como esenciales del oficio (conocimientos diversos necesarios para la conducción de la secuencia de clase) y la personalidad de quien enseña (Vinatier, 2013).

\subsection{El autorreflexión de la práctica docente como dispositivo de formación continua}

El mayor desafío para la persona docente es reconocer lo que sucede en el aula de clases. El hecho de que quien enseña se encuentre en el aula no resulta suficiente para que sepa lo que sucede, ya que las funciones que ha de cumplir propician un estado de repetición de aquellas acciones que gracias a la experiencia ha detectado como útiles. Hacerse sujetos conscientes de la propia práctica en el aula, es el primer paso para realizar acciones para mejorarla. (Vinatier, 2013).

El modelo E-P-R actúa como un dispositivo de coexplicitación de la práctica. Este dispositivo de coexplicitación funciona en medida que el observador de la práctica docente explicita o hace evidente el trabajo docente en el aula. Pastré (2011) expuso que los dispositivos de coexplicitación tienen el objetivo de generar una autorreflexión en la persona docente, la cual permite detectar las fortalezas y las áreas de oportunidad con las que cuenta.

Fierro y Fortoul (2015) plantearon que la reflexión sobre la propia práctica facilita:

a) la ampliación de su conceptualización de lo educativo.

b) el reforzamiento de su poder de actuación (agencia) en tres sentidos: poder resignificar, poder actuar y poder plantearse horizontes de futuro (Gómez, 2015). 
c) la posibilidad de nombrar y categorizar problemáticas áulicas al contar con una herramienta conceptual (el modelo interaccionista de la práctica docente) (Fierro y Fortoul, 2015, p. 8).

Asimismo, Espejo (2017) rescata la importancia del pensamiento reflexivo, que es definido como una meditación constante y cuidadosa, donde se contrastan las propias creencias, ideas y conocimientos con aquellos planteamientos teóricos que los sustentan: "se trata entonces de considerar que la reflexión es una condición sine qua non del desarrollo profesional docente" (Dewey, 1910 citado en Espejo, 2017, p. 54).

De la misma manera, Espejo (2017) explica que para que el docente en servicio piense de forma reflexiva deben de estar presentes cuatro aspectos: (1) voluntad para pensar de forma reflexiva, (2) tiempo necesario para hacerlo, (3) volver a la situación sobre la que se reflexiona las veces que sea necesario, y (4) hacerlo de forma sistematizada. A partir de estos aspectos puede visualizarse que pensar de forma reflexiva no puede ser un acto improvisado, sino que debe ser un ejercicio planeado y normalizado para que pueda tener los efectos deseados.

La reflexión sobre la propia práctica puede darse a partir de registros de observación de aula, por medio de dispositivos de video y audio. Una vez que el docente cuenta con los registros de su práctica puede entrar en un ejercicio de autoobservación y de reflexión acerca de su práctica en el aula. El discurso que se deriva de este tipo de ejercicio reflexivo sobre la práctica docente no es de tipo normativo, es decir, no se orienta a contrastar los supuestos que provienen de la didáctica de las ciencias, con los desempeños docentes, no se propone evidenciar errores de su práctica. Tiene como finalidad conocer y hacer evidente cómo interpreta cada docente el conocimiento y la realidad en la que se encuentra inmiscuido, es decir sus lógicas de actuación y los principios que las sustentan. En consecuencia, se trata de un dispositivo de formación que descansa en el reconocimiento, de parte de los sujetos, de aquellos elementos que conectan sus propósitos y actuaciones con las de sus estudiantes, dando lugar a cuestionamientos que consecuentemente serán un poderoso motor para la autoformación y la mejora continua.

Además, reflexionar sobre la práctica aporta un dispositivo de formación que permite al y a la docente valorar su propia actuación en aula. Espejo (2017, p. 53) plantea que "uno de los aspectos más importantes de la formación en servicio, incluyendo el caso de los profesores universitarios, es la denominada práctica reflexiva". La propia práctica es un 
motor externo que aporta aprendizaje al docente, ya que le permite visualizarse, es decir, verse en un espejo. A través de la autoobservación, la persona docente es capaz de ver las características de su práctica y por lo tanto puede mejorarla. La oportunidad de poder descubrir quiénes somos como enseñantes es un elemento fundamental para la formación docente (Perrenoud, 2007; Schön, 1998).

Bélair (2010), expuso que la formación reflexiva docente implica una necesidad y ejercicio permanente de autoevaluación y cuestionamiento sobre las propias acciones que se llevan a cabo en el aula, lo que se vuelve necesario para comprender el significado que estas acciones tienen y profundizar en cómo y cuándo estas acciones han adquirido tales significados. En este sentido, la formación continua puede darse en estos espacios donde la reflexión sobre el ser y el hacer se muestran explícitos ante quien enseña, y como si esto fuese obra de alguien más, la persona docente puede verse a sí misma y entender que gracias a su propia complejidad su práctica es única e incomparable.

\section{Conclusiones}

El modelo E-P-R de Vinatier (2013) reconoce que la labor docente es compleja y multifactorial, ya que es influenciada por un conjunto amplio de elementos personales, sociales y políticos. Esta complejidad aumenta, pues los y las docentes se encuentran en una tensión constante al buscar el equilibrio de tres grandes polos de tensión: (1) el polo epistemológico, (2) el polo pragmático y (3) el polo relacional. La persona docente busca el equilibrio entre estos tres polos de tensión cuando, por ejemplo, busca atender el avance de sus programas y contenidos curriculares, así como el progreso del saber en sus estudiantes, y al mismo tiempo busca generar una dinámica de trabajo conjunta y cercana a partir de las interacciones con sus estudiantes.

El modelo E-P-R permite que las personas docentes puedan verse a sí mismas, es decir, que logren hacerse sujetos conscientes acerca de cómo es su práctica y cuáles son las acciones que llevan a cabo en el aula. La oportunidad de hacerse sujetos conscientes les permite reflexionar acerca del por qué hace lo que hace y develar la construcción de sentido que subyace a su forma de desenvolverse. Además, el ejercicio reflexivo da la oportunidad de profundizar en cuestionamientos como: ¿cómo he construido o aprendido las acciones que llevo a cabo?, ¿qué sensaciones o sentires experimento cuando realizo esa acción?, ¿me funciona o no hacer las cosas de esa manera?, ¿qué significado tiene esta acción para mí?, ¿por qué hago las cosas de esta manera y no de otra?, ¿por qué me funciona? ¿qué 
consecuencias tiene el que trabaje de esta manera con mis estudiantes?, ¿qué me sugiere para la reflexión el analizar sus producciones de clase, así como sus desempeños en las distintas tareas? Esta serie de preguntas pueden marcar la pauta para que quien educa mejore su práctica a través de la autorreflexión.

A diferencia de otros modelos ${ }^{6}$ que analizan las prácticas docentes en el aula, el modelo E-P-R da por sentado que las reglas bajo las que se orienta la práctica docente son las que suceden en el aula, es decir, que no se busca comparar la práctica docente con lo que la normativa oficial dice que debería ser. No es así como un o una docente progresa en el desarrollo de su profesionalidad. Al no tener una necesidad de comparar constantemente la práctica docente con lineamientos oficiales o con la práctica de otros docentes se deja de lado el discurso evaluativo que puede surgir a partir de la observación y análisis de las prácticas docentes al margen de las comprensiones de los propios docentes. A la vez, se devuelve el proceso de generar preguntas significativas sobre la mejora de las prácticas a las comunidades de docentes que revisan y analizan de manera crítica y propositiva su trabajo.

A este ejercicio de reflexión en comunidades de docentes, el modelo les aporta un elemento fundamental que proviene del análisis de las producciones del estudiantado, así como de la observación y análisis de sus desempeños en el aula. Las diversas fuentes consideradas para reflexionar lo que las situaciones de aula producen en términos de las figuras de saber a las que hemos aludido —referidas a la apropiación de un saber objeto, al dominio de una actividad, situación u objeto o a la apropiación de dispositivos relacionalesrepresentan la oportunidad de cuestionar y trascender las explicaciones basadas en prejuicios o en ideas de sentido común a propósito de los asuntos analizados y de los problemas que se observan.

Así, el análisis del polo de tensión epistemológico ofrecerá elementos para que las personas docentes reflexionen sobre la manera en que acompañan al estudiantado en su progreso curricular. A la vez, se evidencia la manera en que la agenda "formativa", esto es, la apropiación de formas de trato entre el estudiantado, corre en paralelo con el dominio de

\footnotetext{
${ }^{6}$ Particularmente dos: 1) el método Stallings, que consiste en un instrumento utilizado para observar de forma sistematizada la práctica de la persona docente en el aula. Su finalidad es medir el tiempo efectivo que el profesorado de educación primaria dedica a la construcción de los aprendizajes (Secretaría de Educación Pública, 2011, p. 2) el sistema de observación CLASS (Clasroom Assesment Scoring Sistem, por sus siglas en inglés), que trata de la observación sistematizada de las interacciones docente-estudiante en el aula. Su finalidad es medir la calidad de las interacciones observadas en el aula (Pianta, La Paro y Hamre, 2008).
} 
saberes o la manipulación de objetos. La reflexión desde la dimensión relacional pone de manifiesto cuánto de la vida en las aulas se juega en función de los términos de referencia que las personas docentes establecen con sus estudiantes, así como de la manera de atender las disrupciones, tensiones o conflictos que surgen de la vida compartida. La cercanía, el aprecio, la capacidad de reconocer y valorar las diferencias individuales del estudiantado y de aprovecharlas como recurso pedagógico son, entre otros, asuntos que esta dimensión releva.

Finalmente, la dimensión pragmática que remite la manera de conducir secuencias y actividades, recursos, tiempos y tareas, la cual reúne el tipo de asuntos destacados en la didáctica - por ello más accesible para los y las docentes- toma su justa dimensión al contar con elementos que permiten al cuerpo docente responderse preguntas como ¿qué conecta las intenciones, estructuras y secuencias de la clase planeada con los desempeños observados?

Así, el modelo E-P-R, más que orientarse a identificar lo correcto o no de las prácticas, busca generar en la persona docente un sentir de apertura, de ser comprendido, al mismo tiempo que se avanza en la comprensión sobre lo que el estudiantado puede estar experimentando en la clase.

Asimismo, el modelo E-P-R parte de la premisa de que las prácticas docentes no son correctas o incorrectas, sino que lo que sucede en cada aula es único, y que estas son el producto de la influencia de quiénes son los docentes como personas, de sus vivencias y de sus contextos particulares, ya que estos elementos determinan y condicionan sus acciones.

A la vez, el modelo busca que la persona docente visualice su quehacer en el aula como una co-construcción, ya que cada uno de sus estudiantes aportan sus propias vivencias, referentes, biografías y expectativas al espacio de la clase. Tenemos como resultado entonces, el aula como un espacio situado de prácticas, complejo y en permanente co-construcción.

La oportunidad de que las personas docentes aumenten la comprensión crítica y también compasiva sobre el acontecer de aula puede abrir la puerta hacia la mejora de las prácticas, desde una mirada consciente y reflexiva mucho más profunda y por tanto, capaz de dar lugar a procesos de mejora de las prácticas de largo aliento. A pesar de ser un tema escasamente retomado y aplicado en América Latina, en México, se han tenido experiencias formativas con docentes de educación básica que hacen uso del modelo E-P-R, como un referente teórico-metodológico para el análisis de las prácticas docentes en situación (Fierro 
y Fortoul, 2014; 2015; 2016; 2017). Las experiencias realizadas ponen de manifiesto que el modelo puede ser comprendido sin dificultad y utilizado para reflexionar secuencias de clase que resultan de interés para el cuerpo docente, ya sea porque presenta dificultades especiales o por referir a situaciones recurrentes de clase sobre las cuales quisieran reflexionar e introducir formas distintas de manejo.

El modelo fortalece a las personas docentes como sujetos, como profesionales competentes, capaces de desarrollar una mirada autoevaluativa y crítica sobre su práctica y la de sus colegas. Por ello, anima la colegialidad y apuesta por los propios sujetos como los más interesados en vislumbrar y recorrer nuevos senderos que amplíen los horizontes de su quehacer educativo. Los invita a hacer de su profesión una verdadera praxis.

\section{Agradecimientos}

Para la realización de este trabajo se contó con el apoyo de la Beca Nacional brindada por el Consejo Nacional de Ciencia y Tecnología (CONACyT) y los estudiantes de posgrado que cursan programas dentro del Padrón Nacional de Posgrados de Calidad (PNPC).

\section{Referencias}

Bélair, Louise. (2010). Enseñar a complejidad del oficio de maestro. En Leopold Paquay, Marguerite Altet, Evelyne Charlier, y Philippe Perrenoud (Coords), La formación profesional del maestro. Estrategias y competencias (pp. 68-105). Estado de México, México: Fondo de Cultura Económica.

Berger, Peter y Luckmann, Thomas. (1968). La construcción social de la realidad. Buenos Aires, Argentina: Amorrortu.

Blumer, Herbert. (1969). Symbolic Interactionism. Perspective and method. California, Estados Unidos de América: University of Caifornia Press.

Brousseau, Guy. (2007). Iniciación al estudio de la teoría de las situaciones didácticas. Buenos Aires, Argentina: Zorzal.

De Lella, Cayetano. (1999). Modelos y tendencias de la formación docente. Recuperado de https://www.oei.es/historico/cayetano.htm

Espejo, Roberto. (2017). Aprendiendo de la experiencia: un dispositivo de análisis colaborativo de experiencias pedagógicas. Perspectiva Educacional. Formación de profesores, 56(2), 51-69. Recuperado de http://www.perspectivaeducacional.cl/index.php/peducacional/article/viewFile/525/241 
Fierro, Cecilia y Fortoul, Bertha. (2014). La gestión de los aprendizajes para la convivencia y para las disciplinas en las prácticas docentes, como objeto de estudio. En Cristina Perales, Eduardo Arias y Miguel Bazdresch (Coords.), Desarrollo socioafectivo y convivencia escolar (pp. 61-89). Jalisco, México: ITESO.

Fierro, Cecilia y Fortoul, Bertha. (2015). Un modelo interaccionista de análisis de prácticas para la formación de docentes. Memoria del XIII Congreso Nacional de Investigación Educativa. Chihuahua, México.

Fierro, Cecilia y Fortoul, Bertha. (2017). Entretejer espacios para aprender y convivir en el aula. Estado de México, México: Ediciones SM.

Fortoul, Bertha y Fierro, Cecilia. (2016). Rostros de la inclusión en prácticas docentes situadas en entornos vulnerables. En Irazema Ramírez (Comp), Voces de la inclusión. Interpelaciones y críticas a la idea de "inclusión" escolar (pp. 228-263). Veracruz, México: Praxis editorial.

Gómez, Elba. (2015). Agentes y lazos sociales. La experiencia de volverse comunidad. Jalisco, México ITESO.

Hernández, Gerardo. (2012). Paradigmas en psicología de la educación. Barcelona, España: Paidos Educador.

Moscato, Patricia. (2016). La entrevista de autoconfrontación como un dispositivo facilitador de aprendizaje y mejora en la formación docente. En Ana Pereyra, Patricia Moscato, Liliana Calderón y María Oviedo (Eds.), Análisis de las prácticas docentes desde la didáctica profesional (pp. 27-38). Buenos Aires, Argentina: UNIPE.

Paquay, Leopold, Altet, Marguerite, Charlier, Evelyne y Perrenoud, Philippe. (2010). La formación profesional del maestro. Estrategias y competencias. Estado de México, México: Fondo de Cultura Económica.

Pastré, Pierre. (2002). L'analyse du travail en didactique professionnelle [Análisis de la práctica desde la didáctica profesional]. Revue Française de Pédagogie, (138), 9-17. Recuperado de http://www.persee.fr/doc/rfp 0556-7807 2002 num 13812859

Pastré, Pierre. (2006). Apprendre à faire [Aprender a hacer]. En Bourgeois, Etienne y Chapelle, Gaëtane (Eds.). Apprendre et faire apprendre [Aprender y hacer aprender]. (pp. 109-121). Paris, Francia: PUF.

Pastré, Pierre. (2007). Quelques réflexions sur l'organisation de l'activité enseignante. Recherche \& Formation, 56, 81-93. Recuperado de http://rechercheformation.revues.org/907

Pastré, Pierre. (2011). La didactique professionnelle. Approche anthropologique du développement chez les adultes [Didáctica profesional Enfoque antropológico del desarrollo en adultos]. Recherche \& Formation, 179, 138-141. Recuperado de https://rfp.revues.org/3730 
Pereyra, Ana, Moscato, Patricia, Calderón, Liliana y Oviedo, María. (2016). Análisis de las prácticas docentes desde la didáctica profesional. Buenos Aires, Argentina: UNIPE.

Perrenoud, Philippe. (2007). Desarrollar la práctica reflexiva en el oficio de enseñar. Profesionalización y razón pedagógica. Barcelona, España: Graó/Colofón.

Pianta, Robert, La Paro, Karen y Hamre, Bridget. (2008). Classroom Assessment Scoring System: Manual K-3. Virginia, Estados Unidos de América: Paul H Brookes Publishing.

Schön, Donald. (1998). El profesional reflexivo: ¿cómo piensan los profesionales cuando actúan? Barcelona, España: Paidós.

Secretaría de Educación Pública. (2011). Observación en el salón de clase a partir del sistema de observación Stallings. Manual y guía del usuario. Guía y herramienta para medir el tiempo de enseñanza en clase. Ciudad de México, México: Autor.

Vergnaud, Gerard. (1990). Psicologia Cognitiva Dello Sviluppo E Didattica Della Matematica [Psicología Cognitiva del Desarrollo y Educación Matemática]. Turín, Italia: Politecnico di Torino.

Vinatier, Isabelle y Altet, Marguerite. (2008). Analyser et comprendre la pratique enseignante [Analizar y comprender la práctica docente]. Nantes, Francia: Presses universitaires de Rennes.

Vinatier, Isabelle. (2013). Le travail de l'enseignant. Une approche par la didactique professionnelle [El trabajo del docente. Un acercamiento desde la didáctica profesional]. Paris, Francia: De Boeck.

Vinatier, Isabelle. (2015). Análisis de las interacciones escolares y formación de los docentes. Ponencia presentada en el XIII Congreso Nacional de Investigación. Chihuahua, México. 